\title{
Regulation and Covering Problems in MP Systems
}

\author{
Giuditta Franco, Vincenzo Manca, and Roberto Pagliarini \\ Verona University \\ Computer Science Department \\ Strada Le Grazie 15, 37134 Verona, Italy \\ \{giuditta.franco, vincenzo.manca, roberto.pagliarini\}@univr.it
}

Summary. The study of efficient methods to deduce fluxes of biological reactions, by starting from experimental data, is necessary to understand the dynamics of a metabolic model, but it is also a central issue in systems biology. In this paper we report some partial results and related open problems regarding the efficient computation of regulation fluxes in metabolic P systems. By means of Log-gain theory the system dynamics can be linearized, in such a way to be described by a recurrence equations system, of which we point out a few algebraic properties, involving covering problems.

\section{Introduction}

Since their first introduction [14], P systems have been widely investigated in the framework of formal language theory as innovative compartmentalized multiset rewriting systems [15], and different variants of them were analyzed along with their computational power (for a complete list of references, see http://ppage.psystems.eu/). Although they were originally introduced as computational models, their biologically inspired structure and functioning, together with their feasibility as models of cellular and biomolecular processes, turned out to be a widely applicable modeling technique in several domains, including medicine (for immunological processes [6], and cellular tissue healing [5]), economics [16], linguistics and computer science (computer graphics, cryptography, approximate solutions to optimization problems) [4], and, of course, biology (for mechanosensitive channels [1], respiration in bacteria, photosynthesis, the protein kinase $\mathrm{C}$ activation [3]).

The intent to employ multiset processing in the compartmentalized framework provided by cell-like or tissue-like membrane structures in order to study real biological systems is nowadays vividly pursued, along with variants of $\mathrm{P}$ systems enriched with several other features, usually inspired by biology [2]. An important aspect in modeling biological reactions by rewriting rules was a thorough study of the rule application strategy [7], since the traditional nondeterministic maximally 
parallel way seemed to be not enough realistic. Along this recent more applicative trend, a body of research is focused on the modeling of metabolisms, where the main interest is devoted to the molecular reactions transforming matter rather than to the biochemicals distribution and coordination in compartments. Metabolic $P$ systems have been introduced $[8,10]$ as mono-membrane multiset rewriting grammars, where rules are regulated by specific functions. The goal is to control the matter transformation of a reactor by means of rules whose strength and application depend on the objects population concentration.

Reactions are specified along with dynamical fluxes, and each flux denotes the ability of the corresponding rule to compete against other rules in capturing part of the population on which it is applied [10]. This new strategy of rule application was inspired by real 'metabolic reactions', and it seems to lead multiset based computing towards interesting simulations of biological processes, such as complex oscillations [11], the mitotic cycle [3] and the non-photochemical quenching phenomenon [12]. Overall, a new way to observe the evolution rules of a system reproducing a metabolic reaction was proposed. Indeed, since the application of every rule changes the relative amounts of reacting substances, it was enforced that such quantities influence the reactivity of the rules in a way that their application depends on the current substances concentration, as it normally happens in biochemical phenomena. A simulator (named MetaPlab) applying evolution rules with this strategy has been developed, and employed to simulate several biological processes (it may be downloaded from the website mplab.sci.univr.it/, where also several references are reported).

Before entering in more technical details, let us discuss a few other substantial (for modeling purposes) differences which have been introduced by metabolic P systems with respect to traditional membrane systems.

$\mathrm{P}$ systems are traditionally organized in a way that their evolution is synchronous, i.e., a global clock triggers the production of new symbols inside all membranes. In principle, one may try to increase the granularity of a P system in order to obtain fine-grained sequences of transitions, then consider the trajectories described by these sequences, and this description would be as accurate as fine the granularity of the $\mathrm{P}$ system is. In practice, it is likely that the desired granularity is obtained by adding auxiliary symbols or priority constraints in the system, to form (sometimes complex) priority relationships for the rewriting rules [6]. As a matter of fact, $\mathrm{P}$ systems do not provide tools for controling the resolution of the observation of intermediate states, and they are better suited to model a process as a sequence of "snapshots", each one being taken when no more rewriting rules can be applied. With Metabolic P systems instead, one assumes the a priori choice of a time interval $\tau$, between consecutive observation instants, that depends on the macroscopic level at which considering the dynamics of the biological system. The flux values (also called reaction units) are computed according to the chosen observation granularity.

In metabolic $\mathrm{P}$ systems rules are obviously global and not compartmentalized, and the environment changes are taken into account by the fluxes associated to 
reactions. The state, on which reaction units depend, is given by both the value of some magnitudes, called parameters, which can influence the reactions (e.g., temperature and pressure) and by the amount of the substances inside the system. Some distinction between matter and not matter is fundamental to study metabolic processes, and the idea of considering parameters as elements of the system different from metabolic substrates, and having their own evolution, is new of metabolic P systems. Nevertheless, some similar ideas were formalized in the context of membrane systems, by means of promoters and inhibitors, that are respectively permitting and forbidding objects associated to regions, modeling the chemicals in the cell that, while supporting or forbidding certain reactions, can separately evolve, in parallel with the chemicals involved in the reactions [4]. Finally, we would like to emphasize that the approach of modeling by metabolic $\mathrm{P}$ systems assumes a novel perspective, by considering the rules only as matter transformation reactions rather than precise molecular interactions. The search of fluxes is therefore aimed at designing a model of the observed macroscopic reality with respect to the abstract transformations one has assumed, and it is different from the parameter (or rate) estimation typically studied in systems biology, even in the framework of membrane systems [17].

In the next section the problem we tackle is framed, after a brief introduction to MP systems and the Log-gain theory [9], specifically devised for them. A few results are reported in the third section, while a last section about open problems and ongoing work concludes the paper.

\section{Framing of the problem}

An MP system is completely specified by: i) $m$ reactions, ii) $m$ corresponding flux regulation functions, iii) $n$ substances, which are the elements transformed by reactions, and their initial values, iv) $k$ parameters, which are arguments (beside substances) of flux regulation functions, and v) $k$ parameter evolution functions.

We assume $m>n$ (more rules than substances), as it realistically happens in biochemical systems. A few examples are given by the following protein-protein interaction networks: Ito (yeast) has 8868 known interactions among 3280 proteins, Giot (Drosophila) has 4780 known interactions among 4679 proteins, and Li (C. elegans) has 5534 known interactions among 3024 proteins, and by the following bacterial metabolic networks: Wolbachia pipientis has 8128 interactions over 2100 genes, S. enterica has 13309 interactions over 3717 genes, R. felis has 6966 interactions over 2062 genes, and A. phagocytophilum has 7924 over 2056 genes. By a more abstract perspective, we observe that since usually each metabolic reaction transforms few substances, in the case $m \leq n$ we would have a scarce competition for substances among the rules, and the interaction system would be not interesting to analyze. Finally, the problem we are going to describe would be just not so significant from an algebraic viewpoint. The number $k$ of parameters instead, has no relationship with $m$ and $n$, as it just represents the sensitivity of the system to 
the environment (parameters are internal or external controling variables which somehow affect the system functioning).

A state $S$ is an $\mathbb{R}^{n+k}$ vector (reporting the current amounts of substances and parameters), while each rule $r_{j}$ (with $j=1, \ldots, m$ ) having some of the $n$ substances as substrates and some as products, is associated to a couple of $\mathbb{R}^{n}$ vectors $\left(r_{j}^{-}, r_{j}^{+}\right)$(one of which possibly null), reporting the substance quantities respectively occurring in the premise and in the consequence of $r_{j}$. As an instance, we might have a system $\mathcal{Q}$ with three substances $\{a, b, c\}$, two parameters $\{v, w\}$ which values evolve according with their own function vector $\left(f_{v}(i), f_{w}(i)\right)$, for $i \in \mathbb{N}$, and four rules

$$
r_{1}: a b \rightarrow a a, r_{2}: b c c \rightarrow a, r_{3}: a c \rightarrow \lambda, r_{4}: a b c \rightarrow b b .
$$

The reactions respectively correspond to the vector couples:

$$
\begin{aligned}
& \left(r_{1}^{-}, r_{1}^{+}\right)=((1,1,0),(2,0,0)),\left(r_{2}^{-}, r_{2}^{+}\right)=((0,1,2),(1,0,0)), \\
& \left(r_{3}^{-}, r_{3}^{+}\right)=((1,0,1),(0,0,0)),\left(r_{4}^{-}, r_{4}^{+}\right)=((1,1,1),(0,2,0)) .
\end{aligned}
$$

Furthermore, four (one for each rule) flux regulation functions may be given, defined on $\mathbb{R}^{5}$ and having values in $\mathbb{R}$, in order to have the fluxes $u_{1}, u_{2}, u_{3}, u_{4}$, associated respectively to the rules.

There are a couple of features to point out when dealing with metabolic rules $r$. One is the activation substrate (that is, how many units of substrate are necessary in order that the rule be applied), given by the vector $r^{-}$, and the other one is the effect of the rule application, given by $r^{+}-r^{-}$. This last vector gives the biochemical balance due to the application of the rule, that is how much of each substance was either consumed or produced. For example, in the above rule $r_{4}$, we need to have all $u_{4}$ units of $a, u_{4}$ units of $b$ and $u_{4}$ units of $c$ to activate the rule (i.e., to be able to apply the rule), while the rule effectively producing $u_{4}$ of $b$ and consuming $u_{4}$ of $a$ and of $c$. Of course, in cases of no substance production (as it is for $r_{3}$ in the example), the activation and the consumption of the rule coincide.

We call stoichiometric matrix $R$, the $(n \times m)$-dimensional matrix formed by the vectors $r^{+}-r^{-}$, for every rule $r$, disposed according to some prefixed order (which is not relevant). In the example above, the order is given by the index of the rules, and we have

$$
R=\left(\begin{array}{rrrr}
1 & 1 & -1 & -1 \\
-1 & -1 & 0 & 1 \\
0 & -2 & -1 & -1
\end{array}\right)
$$

The stoichiometric matrix is assumed to have maximal rank, as it is the case in our example. If we would have any row linearly dependent on the others, we could delete it (together with the corresponding substance in the system, as studying its dynamics would be not meaningful), and reset the whole system with the remaining substances (we newly say $n$ ) and the corresponding $n \times m$ stoichiometric matrix (having full rank, after the eventual iteration of this procedure).

Analogously, the activation matrix $A$ is formed by the vectors $r^{-}$, and for the example above we have: 


$$
A=\left(\begin{array}{llll}
1 & 0 & 1 & 1 \\
1 & 1 & 0 & 1 \\
0 & 2 & 1 & 1
\end{array}\right) .
$$

The dynamics of a metabolic $\mathrm{P}$ system is given by both the evolution of parameters, according to their laws, and by the evolution of the vector $X$ of substances, ruled by the following recurrence $n$-equations system [10] (where $\times$ denotes the ordinary matrix product and $i$ the discrete instant of time):

$$
X[i+1]=R \times U[i]+X[i] .
$$

By considering $U[i]$ as the unknown vector, the linear system (2) (called ADA for "Avogadro and Dalton Action" [10]) has infinite solutions, as the number $n$ of equations is usually smaller than the number $m$ of variables (should we have the case $m \leq n$, from an algebraic point of view there would be no problem to eventually solve the system or figure out if there is not any solution).

In [9] the Log-gain theory was developed to design an MP model from observation experimental data, that is, to deduce the MP regulation fluxes from temporal series of the substances. From an algebraic viewpoint, such a theory provides us with other $m$ equations and other $n$ variables, that can be added to the ADA system (2) in order to obtain an $n+m$ equations system univocally solvable.

According to the simplest formulation of this theory, given a number of observation steps (at a specified time interval $\tau$ ), and the corresponding time series of the observed states of a real metabolic system (with an assumed stoichiometry), the relative variations of any reaction flux $u_{j}$ of the rule $r_{j}: \alpha_{j} \rightarrow \beta_{j}(j=1, \ldots, m)$ is the sum of the relative variations of the reactants (i.e., the substances occurring in $\alpha_{j}$ ), apart of some error $p_{j}$, which is introduced as a variable of the system. We denote with $P$ the $m$-dimensional vector of such variables, called reaction offsets [9], that is, the errors introduced in the log-gain approximations of fluxes. Furthermore, we denote with $L g(U[i])$ the $m$-dimentional vector of relative fluctuations, that is $\left(\frac{u_{j}[i+1]-u_{j}[i]}{u_{j}[i]} \mid j=1, \ldots, m\right)$, for any $i \in \mathbb{N}$. Analogously $\operatorname{Lg}(X[i])$ and $L g(S[i])$ are the vectors of relative fluctuations respectively of substances and of both substances and parameters.

In formal terms, the $m+n$ equations system we want to solve (in order to find the vector $\mathrm{U}[\mathrm{i}+1])$ is

$$
\left\{\begin{array}{l}
\operatorname{Lg}(U[i])=B \times \operatorname{Lg}(X[i])+C \cdot P \\
R \times U[i+1]=X[i+2]-X[i+1]
\end{array}\right.
$$

where $B$ is a $(m \times n)$-dimentional boolean matrix selecting, by matrix product, the reactants for each reaction, and $C$ is an $m$-dimensional boolean vector selecting, by entrywise product ${ }^{1}$, only $n$ of the $m$ offsets (that are $n$ unknowns of the system, besides the $m$ fluxes).

${ }^{1}$ For two matrices $\mathrm{A}$ and $\mathrm{B}$ having the same dimensions, the Schur product $C=A \cdot B$ is entrywise defined as $C_{i j}=A_{i j} \cdot B_{i j}$. 
According to a more general formulation of the Log-gain theory [9], the relative variations of any reaction flux $u_{j}$ of the rule $r_{j}: \alpha_{j} \rightarrow \beta_{j}(j=1, \ldots, m)$ is the sum of the relative variations of its tuners, which are both the substances (including the reactants) and the parameters which influence the reaction $r_{j}$. In this general case, the system (3) to solve becomes

$$
\left\{\begin{array}{l}
L g(U[i])=B^{\star} \times \operatorname{Lg}(S[i])+C \cdot P \\
R \times U[i+1]=X[i+2]-X[i+1]
\end{array}\right.
$$

where $B^{\star}$ is an $m \times(n+k)$-dimentional boolean matrix selecting, by matrix product, the tuners for each reaction.

\section{A few results}

Given an MP system of substances $\left\{x_{1}, \ldots, x_{n}\right\}$ and rules $\left\{r_{1}, \ldots, r_{m}\right\}, R(x)$ is defined as the set of all rules involving $x$ either as a reactant or as a product. A set $R_{0}$ of $n$ rules is called covering set if $R(x) \cap R_{0} \neq \emptyset$ for any substance $x$. Consequently, the boolean vector $C$ from the system (3), if selecting offsets of rules of a covering set, is called covering vector.

As a first result, we observe that any set of $\mathbf{n}$ linearly independent rules is a covering. In fact, if we look at the $n$ columns of the stoichiometric matrix, corresponding to $n$ prefixed linearly independent rules, they cannot show a null row (otherwise they would be not linearly independent), then any substance (which corresponds to a row) is involved by at least one of the prefixed rules, and this implies they form a covering set. Let us recall here that a set of $n$ linearly independent rules always exists, because the stoichiometric matrix $\mathrm{R}$ (which columns are represented by the rules) is assumed to have maximal rank.

As a second result, the system (3) may be seen as

$$
\left\{\begin{array}{l}
U[i+1]-C \cdot U[i] \cdot P[i+1]=(B \times L g(X[i])) \cdot U[i]+U[i] \\
R \times U[i+1]=X[i+2]-X[i+1]
\end{array}\right.
$$

More interestingly, system (5) may be transformed in another one (see equation (6)) computing the same flux values $U$ by applying a time constant block matrix in each step. The idea underlying this algebraic manipulation is to change the "fake" variables of the system (i.e., those introduced by Log-gain theory in order to be able to solve the linear system (2)), from the $m$-dimensional vector $P[i+1]$ into the $n$-dimensional vector $Z[i+1]$ obtained by taking the $n$ non-null components of $C \cdot U[i] \cdot P[i+1]$. Hence, if we consider as a variable the vector $\left(\begin{array}{c}U[i+1] \\ Z[i+1]\end{array}\right)$ rather than $\left(\begin{array}{c}U[i+1] \\ P[i+1]\end{array}\right)$ we get the following normalized system 


$$
\left(\begin{array}{cc}
I_{m} & G \\
R & O_{n}
\end{array}\right) \times\left(\begin{array}{c}
U[i+1] \\
Z[i+1]
\end{array}\right)=\left(\begin{array}{c}
(B \times L g(X[i])) \cdot U[i]+U[i] \\
X[i+2]-X[i+1]
\end{array}\right)
$$

where $I_{m}$ is the identity matrix of dimension $m, O_{n}$ is the null matrix of dimension $n, R$ is the stoichiometric matrix, and $G$ is an $(m \times n)$-dimensional boolean matrix, called covering matrix, such that each column has exactly one nonnull element and the sum of the first $h$ columns (for any $h=1, \ldots, n$ ) coincide with the covering vector $C$ in its first components containing $h$ ones. In other words, if the non-null components of $C$ are $j_{1}, j_{2}, \ldots, j_{n}$, then the non-null components of the corresponding covering matrix $G$ are $\left(j_{1}, 1\right),\left(j_{2}, 2\right), \ldots,\left(j_{n}, n\right)$.

The systems (5) and (6) are equivalent on the first $m$ components of the unknown vector to compute (i.e., the fluxes we are looking for), because it holds that $G \times Z[i+1]=C \cdot U[i] \cdot P[i+1]$.

Let us see all of this on the example $\mathcal{Q}$ introduced in the previous section. In the stoichiometric matrix $\mathrm{R}$ reported in (1), one can verify that $R_{0}=\left\{r_{1}, r_{2}, r_{3}\right\}$ is a covering set. Then the $7 \times 7$ system (6) to solve is

$$
\left(\begin{array}{cc}
I_{4} & G \\
R & O_{3}
\end{array}\right) \times\left(\begin{array}{c}
U[i+1] \\
Z[i+1]
\end{array}\right)=\left(\begin{array}{c}
(B \times(L g X[i])) \cdot U[i]+U[i] \\
X[i+2]-X[i+1]
\end{array}\right)
$$

where $G=\left(\begin{array}{lll}1 & 0 & 0 \\ 0 & 1 & 0 \\ 0 & 0 & 1 \\ 0 & 0 & 0\end{array}\right), B=\left(\begin{array}{lll}1 & 1 & 0 \\ 0 & 1 & 1 \\ 1 & 0 & 1 \\ 1 & 1 & 1\end{array}\right)$, and $\operatorname{LgX}[i]=\left(\begin{array}{c}\operatorname{Lg}(a[i]) \\ \operatorname{Lg}(b[i]) \\ \operatorname{Lg}(c[i])\end{array}\right)$.

System (6) is a normalized form of the general problem (of finding a linear system to compute the system fluxes), that has been helpful to perform fast and efficient computations for our simulations. Indeed, we do not need to compute the matrix at every computational step as for the system (3), but just once, and the blockwise matrix product can be easily performed by involving operations only on the submatrices.

As a third important result, we can see that, if the matrix $\mathbf{G}$ is defined by a linearly independent covering, then the system (6) has a unique solution. To prove this fact, it is enough to show that the matrix $\mathcal{N}=\left(\begin{array}{cc}I_{m} & G \\ R & O_{n}\end{array}\right)$ has a non-null determinant. Indeed, since it can be written in the following form

$$
\left(\begin{array}{cc}
I_{m} & G \\
R & O_{n}
\end{array}\right)=\left(\begin{array}{cc}
I_{m} & O_{m \times n} \\
R & I_{n}
\end{array}\right) \times\left(\begin{array}{cc}
I_{m} & G \\
O_{n \times m} & -R \times G
\end{array}\right)
$$

then, $\operatorname{det}(\mathcal{N})=-\operatorname{det}(R \times G)$. This implies an even stronger result, that is, the system (6) is univocally solvable if and only if $G$ corresponds to a linearly independent covering.

Since in our recurrence system we are assuming to know the fluxes computed at the previous step, the reader could wonder about the value of the fluxes at 
the initial observation step. There exists a heuristic algorithm to estimate it, by evaluating, along with few steps of observation, how much of each substance is necessary to activate the first evolution step [13].

As a last result, we would like to point out that, the systems (3) and (4) are equivalent (that is, they give the same fluxes), for any $C$ corresponding to a linearly independent covering, if the rows of $B^{\star}$ corresponding to the zero components of $C \cdot P$ have the last $k$ components equal to zero and coincide with the related rows of $B$ in their first $n$ components. In other words, the solution $U$ of the systems (3) and (4) is the same if the fluxes of the non-covered rules are assumed to depend only on the reactants of the rule. Analogously, the solution does not chance if the covered rules have log-gains of the fluxes given by the sum of log-gains not only of the reactants, but also of other elements (substances or parameters).

To prove this, once we have chosen a linearly independent covering $R_{0}$, we arrange the rules of the system according to an order which disposes first the rules of the covering and then the others, so that the stoichiometric matrix $R$ has the first $n$ columns corresponding to the vectors $r^{+}-r^{-}$, for the rules $r \in R_{0}$, and the others to the vectors $r^{+}-r^{-}$, for $r \notin R_{0}$. We denote this feature with the blockwise stoichiometrix matrix $R=\left(R_{0} R_{1}\right)$, where $R_{0}$ is an $n \times n$ matrix, while $R_{1}$ is an $n \times(m-n)$ matrix. The vectors $U=\left(U_{0}, U_{1}\right), C=\left(C_{0}, C_{1}\right)$, and $P=\left(P_{0}, P_{1}\right)$ turn out to be arranged consistently, while $C_{0}$ having all the components equal to one and $C_{1}$ being an $(m-n)$-dimensional null vector. Namely, in the system (4), $B^{\star}=\left(\begin{array}{c}B_{0}^{\star} \\ B_{1}^{\star}\end{array}\right)$ where $B_{0}^{\star}$ is a $n \times(n+k)$ boolean matrix selecting the tuners of each reaction from the matrix $R_{0}$, and $B_{1}^{\star}$ is an $(m-n) \times(n+k)$ boolean matrix selecting the tuners of the reactions from $R_{1}$. Under our hypothesis, if $B=\left(\begin{array}{c}B_{0} \\ B_{1}\end{array}\right)$, with $B_{0}$ and $B_{1}$ respectively $n \times n$ and $(m-n) \times n$-dimensional matrices, then $B_{1}^{\star}=\left(B_{1} O_{(m-n) \times k}\right)$.

If we consider the system (4) in its reformulation (5), we can see

$$
\left\{\begin{array}{l}
U_{0}[i+1]=\left(\left(B_{0}^{\star} \times \operatorname{Lg}(S[i])\right)+1+C_{0} \cdot P_{0}[i+1]\right) \cdot U_{0}[i] \\
U_{1}[i+1]=\left(\left(B_{1}^{\star} \times \operatorname{Lg}(S[i])\right)+1+C_{1} \cdot P_{1}[i+1]\right) \cdot U_{1}[i] \\
R_{0} \times U_{0}[i+1]=X[i+2]-X[i+1]-R_{1} \times U_{1}[i+1]
\end{array}\right.
$$

At this point, we can just notice that, from the second equation (since $C_{1}$ is a null vector and we know the vectors $L g(S[i])$ and $U[i]$ ) we deduce (equivalently in the two systems (3) and (4))

$$
U_{1}[i+1]=\left(B_{1}^{\star} \times L g(S[i])\right) \cdot U_{1}[i]+U_{1}[i]=\left(B_{1} \times L g(X[i])\right) \cdot U_{1}[i]+U_{1}[i] .
$$

On the other hand, since $R_{0}$ has a non-null determinant, the third equation of system (7) has a unique solution for $U_{0}$ (fluxes of covered rules), and it does not depend on the matrix $B_{0}$ but only on $U_{1}[i+1]$. The matrix $B_{0}$ indeed selects only reactants for each rule of the covering $R_{0}$, while here we get the same values 
for both $U_{0}$ and $U_{1}$ even if there is only $B_{0}^{\star}$ involved (by the first equation of the system (7)).

At this point, we can easily see that an even stronger results holds (and it can be similarly proved): the system (4) gives the same values for the fluxes, by keeping constant the choice of the tuners for the non-covered rules, and arbitrarirly modifying the choice of the set of tuners for the covered rules. This observation points out the importance of the covering choice: one essentially selects the rules which are not so important for the dynamics, because it does not really matter which are the substances or parameters which affect them.

An interesting consequence is that the $m$ fluxes can be computed simply solving the $m$ equations system given by

$$
\left\{\begin{array}{l}
\operatorname{Lg}(Y[i])=B^{\star} \times \operatorname{Lg}(S[i]) \\
R \times U[i+1]=X[i+2]-X[i+1]
\end{array}\right.
$$

where $B^{\star}$ is an $(m-n) \times(n+k)$-dimentional boolean matrix selecting, by matrix product, the tuners for each non-covered reaction, and $L g(Y[i])$ is the vector of relative fluctuations of the $m-n$ fluxes corresponding to the non-covered rules.

\section{Future work}

Along the results presented in this paper, MP systems clearly give an exciting connection between linear algebra and rewriting rules, especially those covering substances transformed within a metabolic system, with several facets that require further research. What linear independence of rules means in terms of the biological dynamics, and what in terms of formal rewriting systems? As one of the referees observed, "it would be interesting to determine the meaning of linear independence of rules in the frameworks of both biology and rewriting P systems".

In order to compute fluxes of MP systems, that need to be positive in order rules be applied, it is still not clear which would be the choice of a good covering (among the linearly independent ones). Namely, it seems that conditions on our data may be found that guarantee the positivity of the system fluxes.

Other similarly interesting problems could be outlined if we consider the covering set composed by rules involving all the substances only along their premises. A new formulation of our problem would replace the stoichiometric matrix $R$ with the activation matrix $A$, and it would be interesting to investigate the "more restrictive" conditions we should have from the data to guarantee a "correct" (i.e., with positive fluxes) biological dynamics.

\section{References}

1. I. Ardelean, D. Besozzi, M. H. Garzon, G. Mauri, S. Roy. P System Models for Mechanosensitive Channels, chapter 2 of [4]:43-80. 
2. D. Besozzi, P. Cazzaniga, G. Mauri, D. Pescini. Modelling Metapopulations with Stochastic Membrane Systems, Biosystems 91, 499 - 514, 2008.

3. L. Bianco, F. Fontana, G. Franco, V. Manca. P Systems for Biological Dynamics, chapter 3 of [4], 81-126.

4. G. Ciobanu, M. J. Pérez-Jiménez, Gh. Păun eds. Applications of Membrane Computing, Natural Computing Series, Springer-Verlag New York, Inc., Secaucus, NJ, USA, 2006.

5. G. Franco, N. Jonoska, B. Osborn, A. Plaas. Knee Joint Injury and Repair Modeled by Membrane Systems, BioSystems 91:473-488, 2008.

6. G. Franco, V. Manca. A Membrane System for the Leukocyte Selective Recruitment, C. Martn-Vide et al. eds.: WMC 2003, LNCS 2933, 181 - 190, 2004.

7. M. A. Gutiérrez-Naranjo, Mario J. Pérez-Jiménez, Agustin Riscos-Núñez. On the Degree of Parallelism in Membrane Systems, Theoretical Computer Science 372(23):183-195, 2007.

8. V. Manca. Fundamentals of Metabolic P Systems, In G. Păun et al. eds., chapter 16 of Handbook of Membrane Computing, Oxford University Press, 2009.

9. V. Manca. Log-Gain Principles for Metabolic P Systems, In A. Condon et al. eds., chapter 28 of Algorithmic Bioprocesses, Natural Computing Series, Springer-Verlag, Berlin Heidelberg, 2009.

10. V. Manca. The Metabolic Algorithm for P systems: Principles and Applications, Theoretical Computer Science 404:142-157, 2008.

11. V. Manca, L. Bianco, F. Fontana. Evolution and Oscillation in P systems: Applications to Biological Phenomena, LNCS 3365, 63-84, 2005.

12. V. Manca, R. Pagliarini, S. Zorzan. A Photosynthetic Process Modelled by a Metabolic P System, Natural Computing, 2009. To appear, DOI 10.1007/s11047008-9104-x.

13. R. Pagliarini, G. Franco, V. Manca. An Algorithm for Initial Fluxes of Metabolic P Systems, Int. J. of Computers, Communications \& Control, IV (3): 263-272, 2009.

14. Gh. Păun. Computing with Membranes, Journal of Computer and System Sciences 61(1):108-143, 2000, and Turku Center for Computer Science-TUCS Report 208, November 1998, www.tucs.fi.

15. Gh. Păun. Membrane Computing: An Introduction. Springer, 2002.

16. Gh. Păun, R. A. Păun. Membrane Computing as a Framework for Modeling Economic Processes, Proceedings of the Seventh International Symposium on Symbolic and Numeric Algorithms for Scientific Computing, 11-18, 2005.

17. F. J. Romero-Campero, H. Cao, M. Camara, N. Krasnogor. Structure and Parameter Estimation for Cell Systems Biology Models, Proc. of the Genetic and Evolutionary Computation Conference, July 12-16, Atlanta, USA, 331- 338, 2008. 